\title{
Analyticity and Causality of the Three-Parameter Rheological Models
}

\author{
Nicos Makris ${ }^{\mathrm{a}}$ and Georgios Kampas \\ Department of Civil Engineering, Division of Structures \\ University of Patras, 26500, Greece
}

\begin{abstract}
In this paper the basic frequency response and time response functions of the threeparameter Poynting-Thomson solid and Jeffreys fluid are revisited. The two rheological models find application in several areas of rheology, structural mechanics and geophysics. The relation between the analyticity of a frequency response function and the causality of the corresponding time-response function is established by identifying all singularities at $\omega=0$ after applying a partial fraction expansion to the frequency response functions. The strong singularity at $\omega=0$ in the imaginary part of a frequency response function in association with the causality requirement, imposes the addition of a Dirac delta function in the real part in order to make the frequency response function well defined in the complex plane. This external intervention, which was first discovered by P.A.M. Dirac, has not received the attention it deserves in the literature of viscoelasticity and rheology. The addition of the Dirac delta function makes possible the application of time domain techniques that do not suffer from violating the premise of causality.
\end{abstract}

\section{Introduction}

Traditionally, the linear theory of viscoelasticity has evolved in an inductive manner starting from the "elastic spring" (Hookean Solid) and the "viscous dashpot" (Newtonian fluid) and proceeding to more comprehensive phenomenological models by linear combinations of the two aforementioned basic elements. The behavior of several isotropic materials when stressed at small deformations gradients can be satisfactorily described with combinations of "elastic springs" and "viscous dashpots" and it can be described by linear differential equations with constant coefficients of the form, 


$$
\left[\sum_{m=0}^{M} \alpha_{m} \frac{d^{m}}{d t^{m}}\right] \tau(t)=\left[\sum_{n=0}^{N} b_{n} \frac{d^{n}}{d t^{n}}\right] \gamma(t)
$$

where $\tau(t)$ and $\gamma(t)$ are the time histories of the stress and the small-gradient strain; $\alpha_{m}$ and $b_{m}$ are restricted to real numbers and are the parameters of the constitutive model, while the order of differential $\mathrm{m}$ and $\mathrm{n}$ is restricted to integers. In this paper we are concerned with the integral representation of equation (1). Linear-viscoelastic materials obey the so-called Boltzmann superposition principle- that the output history can be obtained as the convolution of the input history after being convolved with the corresponding basic time-response function. The basic time-response functions can be obtained either by imposing an impulse or a unit-step excitation in the constitutive model or by inverting in the time domain the corresponding frequency response functions of the constitutive models. Such techniques are well known in the disciplines of rheology (Ferry 1980; Bird et al 1987; Tschoegl 1989), structural mechanics (Harris 1988) and automatic control (Bode 1959), among others.

The causal character of frequency response functions enforces relations between their real and imaginary parts (or their log-amplitude and phase). These relations are known as the Kramers-Kronig relations or merely the Hilbert transform (Booij and Thoone 1982; Fannin et al. 1993; Pritz 2003; Parot and Duparray 2007 and references reported therein).

Despite the significant progress in understanding the microscopic topology of polymeric liquids (Everaers et al. 2004), the Hilbert transform (Kramers-Kronig relations) has always been a unique tool to validate experimental results that establish the real or imaginary parts of viscoelastic response functions (Tschoegl 1989; Caracciolo et al. 2001; Parot and Duperray 2007, among others). It should be noted however that there are some materials such as entangled polymer systems, wherethe mechanical straining leads to a considerable enhancement of their microstructure and in this case the Kramers-Kronig relations might be violated. When however microstructural distortion is dominant over microstructural enhancement the KramersKronig relations are found to apply (Dhont and Wagner 2001). Furthermore, experimental imperfections including plate and edge conditions and loss of material from the test-fixture may distort experimental data which appear to be inconsistent 
with the satisfaction of the Kramers-Kronig relations. Such issues require further elaboration (Vlassopoulos 2006).

More than a decade ago, Makris (1997) noticed that frequency response functions that have a singularity at $\omega=0$ in their imaginary part (this happens to all practical models including the Hookean spring, the Newtonian dashpot, the Kelvin-Voigt and Maxwell models) should be corrected by adding a Dirac delta function in their real part. This operation ensures that the resulting time-response function is causal; and within the context of generalized functions (Lighthill 1989) extends the fundamental relation between analyticity of a frequency response function and the causality of the corresponding time-response function. The presence of this extra Dirac delta function in the frequency response functions, which does not appear in standard vibration handbooks (Harris 1988) or in the literature of rheology, appears indirectly in the time-response functions of simple viscoelastic models that have been postulated by Giesekus (1995). The remarkable intuitive results of Giesekus in the time domain are in direct agreement with the fundamental properties of analytic functions in the frequency domain. First, in this paper it is shown the agreement between the basic time-response function presented by Giesekus (1995) and those obtained by Makris (1997) who required that the real and imaginary parts of their frequency response function should be Hilbert pairs.

Subsequently, the three-parameter Poynting-Thomson solid and the three-parameter Jeffreys fluid are examined and it is shown that they posses strictly proper frequency response functions which however have a singularity at zero. Despite that their frequency response functions have more poles than zeros, the fact that they have a pole at $\omega=0$ in their imaginary part requires the addition of an external Dirac delta function in their real part so that their corresponding time-response functions are causal. The paper concludes with a table which summarizes the basic frequency response and time response functions of the three-parameter solid and fluid models. The correct expressions for the complex viscosity of the Poynting-Thomson solid and the complex compliance of the Jeffreys fluid together with the correct expressions of the corresponding relaxation modulus and retardation fluidity are new, original results in the literature. 


\section{Basic Frequency and Time Response Functions}

The linearity of equation (1) permits its transformation in the frequency domain by using the Fourier transform

$$
\tau(\omega)=\left[G_{1}(\omega)+i G_{2}(\omega)\right] \gamma(\omega)
$$

where $\tau(\omega)=\int_{-\infty}^{+\infty} \tau(t) e^{-i \omega t} d t=\mathcal{F}\{\tau(t)\}$ and $\gamma(\omega)=\mathcal{F}\{\gamma(t)\}$ are the Fourier transforms of the stress and strain histories, respectively, while $G_{1}(\omega)+i G_{2}(\omega)$ is the complex dynamic modulus of the model (Ferry 1980; Bird et al 1987; Gisekus1995)

$$
G(\omega)=G_{1}(\omega)+i G_{2}(\omega)=\frac{\sum_{n=0}^{N} b_{n}(i \omega)^{n}}{\sum_{m=0}^{M} a_{m}(i \omega)^{m}}
$$

$\mathcal{G}(\omega)$ is a basic frequency response function that relates a stress output to strain input. The numerator of the right-hand of (3) is a polynomial of degree $n$ and the denominator of degree m; therefore, $\quad \mathcal{G}(\omega)$ has n zeros and m poles. A frequency response function that has more poles than zeros $(\mathrm{m}>\mathrm{n})$ is called strictly proper and results in a strictly causal time-response function which means that it is zero at negative times and finite at the time origin.

The stress $\tau(t)$ in (1) can be computed in the time domain with the convolution integral

$$
\tau(t)=\int_{-\infty}^{t} q(t-\xi) \gamma(\xi) d \xi
$$

where $q(t)=$ memory function of the model (Bird et al 1987), defined as the resulting stress at time $t$ due to an impulsive strain input at time $\xi(\xi<t)$ and is the inverse Fourier transform of the complex dynamic modulus 


$$
q(t)=\frac{1}{2 \pi} \int_{-\infty}^{+\infty} \mathcal{G}(\omega) e^{i \omega t} d \omega
$$

The inverse Fourier transform given by (5) converges only when $\int_{-\infty}^{+\infty}|G(\omega)| d \omega<\infty$; therefore $q(t)$ exists in the classical sense only when $G(\omega)$ is a strictly proper function $(m>n)$. However, there are case where strictly proper frequency response functions have a pole at zero $\omega=0$, and in this case a special treatment is required in their real part in which an addition of an external Dirac delta function is needed. When the number of poles is equal to the number zeros $(m=n)$, the frequency response function of the model is simply proper and results to a timeresponse function that has a singularity at the time origin because of the finite limiting value of the dynamic stiffness at high frequencies. This means that in addition to the hereditary effects, the model responds instantaneously to a given input. When the number of poles is less than the number of zeros $(m<n)$, the frequency response function of the model is improper (Rohrs et al 1993).

The inverse of the complex dynamic modulus is the complex dynamic compliance (Pipkin 1986)

$$
J(\omega)=J_{1}(\omega)+i J_{2}(\omega)=\frac{1}{G_{1}(\omega)+i G_{2}(\omega)},
$$

which is a frequency response function that relates a strain output to a stress input. From (3) and (6) it is clear that when a phenomenological model has a strictly proper complex modulus it has an improper complex compliance and vise versa. Accordingly, when the causality of a proposed model is a concern it is important to specify what is the input and what is the output.

When the dynamic compliance $J(\omega)$ is a proper frequency response function the strain history $\gamma(t)$ in (1) can be computed in the time domain via convolution integral 


$$
\gamma(t)=\int_{-\infty}^{t} \varphi(t-\xi) \tau(\xi) d \xi
$$

where $\varphi(t)=$ retardation fluidity (Giesekus 1995), defined as the resulting strain history at time $t$ due to an impulsive stress input at time $\xi(\xi<t)$ and it is the inverse Fourier transform of the dynamic compliance.

$$
\varphi(t)=\frac{1}{2 \pi} \int_{-\infty}^{+\infty} J(\omega) e^{i \omega t} d \omega .
$$

In structural mechanics the equivalent of the retardation fluidity is known as the impulse response function, $h(t)$ (Veletsos and Verbic 1974; Makris 1997). Expressions of the retardation fluidity of the Hookean solid, the Newtonian fluid, the Kelvin-Voigt solid and Maxwell fluid have been presented by Giesekus (1995); however, the expression of retardation fluidity of the three-parameter Jeffreys' fluid has not been available in the literature. The lack of this expression was part of the motivation of this paper. Another useful frequency response function of a phenomenological model is the complex viscosity $\eta(\omega)=\eta_{1}(\omega)+i \eta_{2}(\omega)$, which relates a stress output to a strain-rate input

$$
\tau(\omega)=\left[\eta_{1}(\omega)+i \eta_{2}(\omega)\right] \dot{\gamma}(\omega),
$$

where $\dot{\gamma}(\omega)=i \omega \gamma(\omega)=$ Fourier transform of the strain-rate time history. In structural mechanics the equivalent of the complex viscosity at the force-velocity level is known as the impedance function $Z(\omega)=Z_{1}(\omega)+i Z_{2}(\omega)$. For the linear viscoelastic model given by (1) the complex viscosity of the model is

$$
\eta(\omega)=\eta_{1}(\omega)+i \eta_{2}(\omega)=\frac{\sum_{n=0}^{N} b_{n}(i \omega)^{n}}{\sum_{m=0}^{m=M} a_{m}(i \omega)^{m+1}}
$$


The stress $\tau(t)$ in (1) can be computed in the time domain with an alternative convolution integral

$$
\tau(t)=\int_{-\infty}^{t} G(t-\xi) \frac{d \gamma(\xi)}{d \xi} d \xi
$$

where $G(t)$ is the relaxation modulus of the model defines as the resulting stress at the present time, $\mathrm{t}$, for a unit-step displacement at time $\xi(\xi<t)$ and is the inverse Fourier transform of the complex viscosity

$$
G(t)=\frac{1}{2 \pi} \int_{-\infty}^{+\infty} \eta(\omega) e^{i \omega t} d \omega
$$

Expressions for the relaxation modulus $G(t)$, of various simple viscoelastic models are well known in the literature (Bird et al. 1987; Giesekus 1995); however the expression of the relaxation modulus of the three-parameter Poynting-Thomson solid has not been available. This issue is addressed in this paper.

Equation (10) indicates that if the complex dynamic modulus of a model, $G(\omega)$, is a simple proper function, then the complex viscosity of the model $\eta(\omega)$, is a strictly proper function; therefore, the relaxation modulus of the model $G(t)$ is finite; whereas, the memory function $q(t)$ has a singularity at the time origin. The inverse of the complex dynamic viscosity is the complex dynamic fluidity (Giesekus 1995)

$$
\varphi(\omega)=\varphi_{1}(\omega)+i \varphi_{2}(\omega)=\frac{1}{\eta_{1}(\omega)+i \eta_{2}(\omega)}
$$

which is a frequency response function that relates a strain-rate output to a stress input. When the dynamic fluidity is a proper frequency response function the rate-ofstrain history $\dot{\gamma}(t)$ can be computed in the time domain via the convolution integral 


$$
\dot{\gamma}(t)=\int_{-\infty}^{t} \psi(t-\xi) \tau(\xi) d \xi
$$

where $\psi(t)$ is the impulse strain-rate response function defined as the resulting strain-rate output at time $\mathrm{t}$ for an impulsive stress input at time $\xi(\xi<t)$ and is the inverse Fourier transform of the dynamic fluidity

$$
\psi(t)=\frac{1}{2 \pi} \int_{-\infty}^{+\infty} \varphi(\omega) e^{i \omega t} d \omega
$$

\section{The Hidden Dirac delta Function}

The need for the addition of a Dirac delta function in the real part of frequency

response functions, that their imaginary part has a singularity at $\omega=0+i 0$ was discovered by Makris (1997) and is illustrated in this section by examining the simplest phenomenological model- the linear elastic spring. For a Hookean solid equation (1) reduces to

$$
\tau(t)=G \gamma(t)
$$

and from equation (3) the complex dynamic modulus is merely

$$
\mathcal{G}(\omega)=G+i 0
$$

while equation (5) yields that the memory function,

$$
q(t)=G \delta(t-0)
$$

Now, equation (9) suggests that the dynamic viscosity of the Hookean solid (strainrate frequency response function)

$$
\eta(\omega)=\eta_{1}(\omega)+i \eta_{2}(\omega)=\frac{G(\omega)}{i \omega}
$$

and according to equation (19) the dynamic viscosity (impedance) of the Hookean solid is 


$$
\eta(\omega)=0-i \frac{G}{\omega}
$$

The inverse Fourier transform, $\frac{1}{2 \pi} \int_{-\infty}^{+\infty}-\frac{i}{\omega} e^{i \omega t} d \omega=\frac{1}{2} \operatorname{sgn}(t)$, is well known in the literature (Morse and Feshbach 1953) and according to equation (12), given the expression offered by (20), the relaxation function of the Hookean solid assumes the expression

$$
G(t)=\frac{1}{2 \pi} \int_{-\infty}^{+\infty}-i \frac{G}{\omega} e^{i \omega t} d \omega=\frac{1}{2} G \operatorname{sgn}(t)
$$

The reader recognizes that although the complex dynamic viscosity of the Hookean spring, given by (20), is a strictly proper function, the resulting relaxation function is the erroneous non-causal signum function which maintains a finite value along the entire negative time axis. In fact, equation (21) erroneously suggests that the elastic spring will produce as much response at negative times (prior to the excitation) as the response produced following the excitation. The origin of this causality violation is that although the expression of the complex viscosity, $\eta(\omega)$, given by (20) is a strictly proper frequency response function, the real and imaginary parts of $\eta(\omega)$ given by (20) are not Hilbert pairs (Tschoegl 1989). The violation of causality shown by (21) can be cured by requiring the real and imaginary parts (20) to be Hilbert pairs, or in other words to satisfy Kramers-Kronig relations

$$
\eta_{1}(\omega)=-\frac{1}{\pi} \int_{-\infty}^{+\infty} \frac{\eta_{2}(x)}{x-\omega} d x, \eta_{2}(\omega)=\frac{1}{\pi} \int_{-\infty}^{+\infty} \frac{\eta_{1}(x)}{x-\omega} d x
$$

The imaginary part, $-\frac{G}{\omega}$, of $\eta(\omega)$ given by (20), has as Hilbert transform the function, $G \pi \delta(\omega-0)$ - a function that is everywhere zero; yet singular at the static limit. This can be shown immediately from the second relation of (22) after making the change of variables $\xi=x-\omega, d \xi=d x$. 
$\eta_{2}(\omega)=\frac{1}{\pi} \int_{-\infty}^{+\infty} \frac{\pi \delta(x-0)}{x-\omega} d x=\int_{-\infty}^{+\infty} \frac{\pi \delta[\xi-(-\omega+0)]}{\xi} d \xi=\frac{1}{-\omega+0}=\frac{1}{\omega}$

Accordingly, the real part of the complex viscosity of the Hookean spring is $\eta_{1}(\omega)=\pi G \delta(\omega-0)$ (not zero) and the correct value of the complex viscosity of the Hookean spring is not equation (20) but the equation below:

$$
\eta(\omega)=\pi G\left[\delta(\omega-0)-i \frac{1}{\pi \omega}\right]
$$

By inverting back in the time domain equation (24), the correct expression for the relaxation modulus of the Hookean spring is recovered

$$
G(t)=\frac{1}{2 \pi} \int_{-\infty}^{+\infty} \pi G\left[\delta(\omega-0)-i \frac{1}{\pi \omega}\right] e^{i \omega t} d \omega=\frac{1}{2} G[1+\operatorname{sgn}(t)]=G H(t)
$$

where $H(t)$ is the Heaviside step function and the correct causal result is recovered (see Table 1, Giesekus 1995). The above calculation shows that the requirement that the real and imaginary part of the complex dynamic viscosity to be Hilbert pairs imposes the presence of a Dirac delta function in its real part which when transformed in the time domain offers the so much needed unity which lifts the signum function by the necessary amount to convert it to the causal Heaviside function. The presence of a Dirac delta function as the real part of the complex viscosity extends the concept of analyticity to generalized functions and essentially makes the reciprocal function $\frac{1}{\omega}$ well defined in the neighborhood $\omega=0$. The intimate relation between the reciprocal function and the delta function appearing in the right hand side of (24) was first noticed by Dirac (1958).

The final result of equation (25), $G(t)=G H(t)$, has been presented in the paper by Giesekus (1995) who followed faithfully the definition of the relaxation modulus and imposed in the constitutive equation of the Hookean solid, $\tau(t)=G \gamma(t)$ a step strain excitation $\gamma(t)=\gamma H(t)$. In this paper the same result is recovered with 
a rigorous mathematical formulation which emerges from the fundamental relation between the causality of the time response function and the analyticity of the corresponding frequency response functions which herein is extended in the case of generalized functions. This mathematical formulation is further applied in this paper in an effort to compute the basic frequency response functions of the three-parameter solid and fluid models.

\section{Complex Modulus, Compliance, Viscosity and Fluidity of Simple Rheological Models}

\section{Backgroung}

All simple linear rheological models such as the Hookean solid, the Newtonian fluid, the Kelvin-Voigt solid, the Maxwell fluid, together with the three-parameter Poynting-Thomson solid and Jeffreys fluid have either a complex compliance or complex viscosity function with an imaginary term that has a singularity at $\omega=0$. By using the properties of the Hilbert transform and the associated Kramers-Kronig relation, Makris (1997) corrected the frequency response functions of the first four models by adding a delta function in their real part. Table 1 presents the corrected basic frequency response functions and the corresponding time-response functions of the first four elementary rheological models at the stress-strain level. This table was first published by Makris (1997) within the context of structural mechanics at the force-displacement level. The appended Dirac delta functions appearing in the complex viscosity (impedance) of the Hookean and Kelvin-Voigt solids and in the complex compliance (flexibility) of the Newtonian and Maxwell fluids are marked in bold face. Similarly in bold face are marked in the corresponding time response functions the $1 / 2$ values resulting from the Fourier transform of the appended Dirac delta functions, which convert the non-causal signum functions, $\frac{1}{2} \operatorname{sgn}(t)$, into the causal Heaviside functions $=\frac{1}{2}+\frac{1}{2} \operatorname{sgn}(t)=H(t)$. It is remarkable that all causal Heaviside functions, $\frac{1}{2}+\frac{1}{2} \operatorname{sgn}(t)$, appearing in Table 1 of this paper, appear also in the paper by Giesekus (1995); which however, does not addresses their implication to the corresponding frequency response functions. 
TABLE 1. Basic Frequency response Functions and Time-Response Functions of Elementary Rheological Models.

\begin{tabular}{|c|c|c|c|c|}
\hline & Hookean solid & Newtonian fluid & Kelvin-Voigt solid & Maxwell fluid \\
\hline Constitutive equation & $\tau(t)=G \gamma(t)$ & $\tau(t)=\eta \frac{d \gamma(t)}{d t}$ & $\tau(t)=G \gamma(t)+\eta \frac{d \gamma(t)}{d t}$ & $\tau(t)+\lambda \frac{d \tau(t)}{d t}=\eta \frac{d \gamma(t)}{d t}$ \\
\hline Complex Modulus: $G(\omega)$ & $G+i 0$ & $0+i \omega \eta$ & $G+i \omega \eta$ & $\eta\left[\frac{\lambda \omega^{2}}{1+\lambda^{2} \omega^{2}}+i \frac{\omega}{1+\lambda^{2} \omega^{2}}\right]$ \\
\hline Complex Compliance: $J(\omega)$ & $\frac{1}{G}+i 0$ & $\frac{\pi}{\eta}\left[\boldsymbol{\delta}(\boldsymbol{\omega}-\mathbf{0})-i \frac{1}{\pi \omega}\right]$ & $\frac{1}{G}\left[\frac{1}{1+\lambda^{2} \omega^{2}}-i \frac{\omega \lambda}{1+\lambda^{2} \omega^{2}}\right]$ & $\frac{\pi}{\eta}\left[\frac{\lambda}{\pi}+\boldsymbol{\delta}(\boldsymbol{\omega}-\mathbf{0})-i \frac{1}{\pi \omega}\right]$ \\
\hline Complex Viscosity, $\eta(\omega)$ & $\pi G\left[\boldsymbol{\delta}(\boldsymbol{\omega}-\mathbf{0})-i \frac{1}{\pi \omega}\right]$ & $\eta+i 0$ & $\pi G\left[\frac{\lambda}{\pi}+\boldsymbol{\delta}(\boldsymbol{\omega}-\mathbf{0})-i \frac{1}{\pi \omega}\right]$ & $\eta\left[\frac{1}{1+\lambda^{2} \omega^{2}}-i \frac{\omega \lambda}{1+\lambda^{2} \omega^{2}}\right]$ \\
\hline Complex Fluidity, $\varphi(\omega)$ & $0+i \omega \frac{1}{G}$ & $\frac{1}{\eta}+i 0$ & $\frac{1}{G}\left[\frac{\lambda \omega^{2}}{1+\lambda^{2} \omega^{2}}+i \frac{\omega}{1+\lambda^{2} \omega^{2}}\right]$ & $\frac{1}{\eta}[1+i \lambda \omega]$ \\
\hline Memory Function, $q(t)$ & $G \delta(t-0)$ & $\eta \frac{d \delta(t-0)}{d t}$ & $G\left[\delta(t-0)+\lambda \frac{d \delta(t-0)}{d t}\right]$ & $\frac{\eta}{\lambda}\left[\delta(t-0)-\frac{1}{\lambda} e^{-t / \lambda}\right]$ \\
\hline Retardation Fluidity, $\varphi(t)$ & $\frac{1}{G} \delta(t-0)$ & $\frac{1}{\eta}\left[\frac{\mathbf{1}}{\mathbf{2}}+\frac{1}{2} \operatorname{sgn}(t)\right]$ & $\frac{1}{\eta} e^{-t / \lambda}$ & $\frac{1}{\eta}\left[\lambda \delta(t-0)+\frac{1}{2}+\frac{1}{2} \operatorname{sgn}(t)\right]$ \\
\hline Relaxation Modulus, $G(t)$ & $G\left[\frac{1}{2}+\frac{1}{2} \operatorname{sgn}(t)\right]$ & $\eta \delta(t-0)$ & $G\left[\lambda \delta(t-0)+\frac{\mathbf{1}}{\mathbf{2}}+\frac{1}{2} \operatorname{sgn}(t)\right]$ & $\frac{\eta}{\lambda} e^{-t / \lambda}$ \\
\hline Impulse Strain-Rate Response Function, $\psi(t)$ & $\frac{1}{G} \frac{d \delta(t-0)}{d t}$ & $\frac{1}{\eta} \delta(t-0)$ & $\frac{1}{\eta}\left[\delta(t-0)-\frac{1}{\lambda} e^{-t / \lambda}\right]$ & $\frac{1}{\eta}\left[\delta(t-0)+\lambda \frac{d \delta(t-0)}{d t}\right]$ \\
\hline
\end{tabular}


In this paper the same methodology is applied to the three parameter PoyntingThomson solid and Jeffrey fluid. Figure 1 shows schematics of the two models of interest as alternative combinations of springs and dashpots.

\section{The Poynting-Thomson Solid}

With reference to Figure 1 (left) the constitutive equation of the three parameter Poynting-Thomson solid is

$$
\tau(t)+\lambda_{1} \frac{d \tau(t)}{d t}=G\left[\gamma(t)+\lambda_{2} \frac{d \gamma(t)}{d t}\right]
$$

For the top configuration of Figure $1 \lambda_{1}=\frac{\eta}{G_{1}+G_{2}}=$ relaxation time,

$\lambda_{2}=\frac{\eta}{G_{2}}=$ the retardation time $\left(\lambda_{2}>\lambda_{1}\right)$ and $G=\frac{G_{1} G_{2}}{G_{1}+G_{2}} ;$ while for the

bottom configuration of Figure $1, \lambda_{1}=\frac{\eta}{G_{2}}, \lambda_{2}=\eta \frac{G_{1}+G_{2}}{G_{1} G_{2}}$ and $G=G_{1}$.

The complex modulus of the Poynting-Thomson solid is

$$
G(\omega)=G \frac{1+i \omega \lambda_{2}}{1+i \omega \lambda_{1}}=\frac{G}{\lambda_{1}}\left(\lambda_{2}-\frac{\lambda_{2}-\lambda_{1}}{1+i \omega \lambda_{1}}\right)
$$

which is a simple proper transfer function since the number of poles equals the numbers of zeros. In the right hand side of equation (29) we have separated the finite limiting value of the complex dynamic modulus at the high frequency limit which when transferred in the time domain it yields a singularity at the time origin of the memory function

$$
q(t)=\frac{1}{2 \pi} \int_{-\infty}^{+\infty} G(\omega) e^{-i \omega t} d \omega=\frac{G}{\lambda_{1}}\left[\lambda_{2} \delta(t-0)-\left(\frac{\lambda_{2}}{\lambda_{1}}-1\right) e^{-\frac{t}{\lambda_{1}}} H(t)\right]
$$

Similarly, the complex dynamic compliance of the Poynting-Thomson model is a simple proper transfer function 


$$
J(\omega)=\frac{1}{G(\omega)}=\frac{1}{G} \frac{1+i \omega \lambda_{1}}{1+i \omega \lambda_{2}}=\frac{1}{G \lambda_{2}}\left(\lambda_{1}+\frac{\lambda_{2}-\lambda_{1}}{1+i \omega \lambda_{2}}\right)
$$

and the retardation fluidity also exhibits a feeble singularity at the time origin.

$$
\varphi(t)=\frac{1}{2 \pi} \int_{-\infty}^{+\infty} J(\omega) e^{i \omega t} d \omega=\frac{1}{G \lambda_{2}}\left[\lambda_{1} \delta(t-0)+\left(1-\frac{\lambda_{1}}{\lambda_{2}}\right) e^{-\frac{t}{\lambda_{2}}}\right]
$$

The complex viscosity of the Poynting-Thomson solid is

$$
\eta(\omega)=\frac{G(\omega)}{i \omega}=G \frac{1+i \omega \lambda_{2}}{i \omega\left(1+i \omega \lambda_{1}\right)}
$$

which is a strictly proper function since the number of poles is larger than the number of zeros. At the same time, the complex viscosity given by (33) has a singularity at $\omega=0$ and a special treatment is required. Partial fraction expansion of the polynomial ratio of equation (33) yields

$$
\eta(\omega)=G\left(\lambda_{2}-\lambda_{1}\right)\left[\frac{1}{1+\omega^{2} \lambda_{1}^{2}}-i \frac{\omega \lambda_{1}}{1+\omega^{2} \lambda_{1}^{2}}\right]-i G \frac{1}{\omega} .
$$

The quantity within the brackets of equation (34) is merely the complex dynamic viscosity of the Maxwell element (see Table 1) and when transformed in the time domain it yields a causal response, $\exp \left(-t / \lambda_{1}\right)$, since the real and imaginary parts in the brackets of (34) are Hilbert pairs (Makris 1997). What remains in the frequency response function of (34) is the last term, $-i G \frac{1}{\omega}$, which is merely the uncorrected $\omega$ complex viscosity of the Hookean solid (see equation 20). Its Hilbert transform is offered by equation (24) and the correct expression for the complex viscosity of the Poynting-Thomson's model is

$$
\eta(\omega)=G\left(\lambda_{2}-\lambda_{1}\right)\left[\frac{1}{1+\lambda_{1}^{2} \omega^{2}}-i \frac{\omega \lambda_{1}}{1+\lambda_{1}^{2} \omega^{2}}\right]+\pi G\left[\delta(\omega-0)-i \frac{1}{\pi \omega}\right]
$$


With the addition of the Dirac delta function, $\pi G \delta(\omega-0)$, in (34) the real and imaginary parts within brackets of equation (35) are now Hilbert pairs and the inverse Fourier transform of (35) yields a relaxation modulus that is causal

$$
G(t)=G\left[\frac{1}{2}+\frac{1}{2} \operatorname{sgn}(t)\right]+G\left(\frac{\lambda_{2}}{\lambda_{1}}-1\right) e^{-\frac{t}{\lambda_{1}}}
$$

The complex dynamic fluidity of the Poynting-Thomson solid is an improper frequency response function

$$
\varphi(\omega)=\frac{1}{\eta(\omega)}=\frac{1}{G} \frac{i \omega\left(1+i \omega \lambda_{1}\right)}{1+i \omega \lambda_{2}}
$$

and its impulse strain-rate response function is computed with equation (15). The inverse Fourier transform of (37) yields the impulse strain-rate response function that is singular at the time origin.

$$
\psi(t)=\frac{1}{G \lambda_{2}}\left[\lambda_{1} \frac{d \delta(t-0)}{d t}+\left(1-\frac{\lambda_{1}}{\lambda_{2}}\right) \delta(t-0)-\frac{1}{\lambda_{2}}\left(1-\frac{\lambda_{1}}{\lambda_{2}}\right) e^{-\frac{t}{\lambda_{2}}}\right]
$$

Table 2 summarizes the basic frequency response and time-response functions of the two models of interest in this paper.

\section{The Jeffreys Model}

With reference to Figure 1 (right) the constitutive equation of the three-parameter Jeffreys fluid is

$$
\tau(t)+\lambda_{1} \frac{d \tau(t)}{d t}=\eta\left[\frac{d \gamma(t)}{d t}+\lambda_{2} \frac{d^{2} \gamma(t)}{d t^{2}}\right]
$$


For the top configuration of Figure $1, \quad \lambda_{1}=\frac{\eta_{1}+\eta_{2}}{G}=$ relaxation time, $\lambda_{2}=\frac{\eta_{2}}{G}=$ retardation time $\left(\lambda_{1}>\lambda_{2}\right)$ and $\eta=\eta_{1} ;$ while, for the bottom configuration of Figure 1, $\lambda_{1}=\frac{\eta_{2}}{G}, \lambda_{2}=\frac{1}{G} \frac{\eta_{1} \eta_{2}}{\eta_{1}+\eta_{2}}$ and $\eta=\eta_{1}+\eta_{2}$.

The Jeffreys fluid has been a popular visoelastic model which has been initially proposed by Jeffreys (1929) to model the viscoelastic behavior of earth strata and subsequently enjoyed wide acceptance by rhelogists in studies ranging from the onset of convection in viscoelastic fluids (Lebon et al. 1994) to the understanding of viscoelastic flow in curved ducts (Manos et al. 2006) and peristaltic transport (Kothandapani and Srinivan 2008).

The complex modulus of the Jeffreys fluid is

$$
G(\omega)=\eta \omega \frac{i-\omega \lambda_{2}}{1+i \omega \lambda_{1}}
$$

which is an improper frequency response function since the number of poles is less than the numbers of zeros and its memory function is computed as

$$
q(t)=\frac{\eta}{\lambda_{1}}\left[\lambda_{2} \frac{d \delta(t-0)}{d t}+\left(1-\frac{\lambda_{2}}{\lambda_{1}}\right) \delta(t-0)-\frac{1}{\lambda_{1}}\left(1-\frac{\lambda_{2}}{\lambda_{1}}\right) e^{-\frac{t}{\lambda_{1}}}\right]
$$

The complex dynamic compliance of the Jeffreys' model is

$$
J(\omega)=\frac{1}{G(\omega)}=\frac{1}{\eta} \frac{1}{\omega} \frac{1+i \omega \lambda_{1}}{i-\omega \lambda_{2}}
$$

which is a strictly proper function; nevertheless, it has a singularity at $\omega=0$ and a special treatment is required. Partial fraction expansion of the polynomial ratio of equation (42) yields 


$$
J(\omega)=\frac{\lambda_{1}-\lambda_{2}}{\eta}\left[\frac{1}{1+\lambda_{2}{ }^{2} \omega^{2}}-i \frac{\omega \lambda_{2}}{1+\lambda_{2}{ }^{2} \omega^{2}}\right]-\frac{i}{\eta} \frac{1}{\omega}
$$

The quantity within the brackets of equation (43) is again the complex dynamic viscosity of the Maxwell element (see Table 1) and when transformed in the time domain it yields a causal response, $\exp \left(-t / \lambda_{1}\right)$, since the real and imaginary parts in the brackets of (43) are Hilbert pairs (Makris 1997). The remaining part in equation (43) is the imaginary quantity, $-\frac{i}{\eta} \frac{1}{\omega}$. Its Hilbert transform is $\frac{\pi}{\eta} \delta(\omega-0)$; and therefore, the correct expression for the complex compliance of the Jeffreys model is

$$
J(\omega)=\frac{\lambda_{1}-\lambda_{2}}{\eta}\left[\frac{1}{1+\lambda_{2}{ }^{2} \omega^{2}}-i \frac{\omega \lambda_{2}}{1+\lambda_{2}{ }^{2} \omega^{2}}\right]+\frac{\pi}{\eta}\left[\delta(\omega-0)-\frac{1}{\pi \omega}\right]
$$

With the addition of the Dirac delta function, $\pi \delta(\omega-0)$, in (43) the real and imaginary part within both parenthesis of equation (44) are Hilbert pairs and the inverse Fourier transform of (44) yields a retardation fluidity function that is causal

$$
\varphi(t)=\frac{1}{\eta}\left[\frac{1}{2}+\frac{1}{2} \operatorname{sgn}(t)+\left(\frac{\lambda_{1}}{\lambda_{2}}-1\right) e^{-\frac{t}{\lambda_{2}}}\right] .
$$

The complex viscosity of the Jeffreys' fluid is

$$
\eta(\omega)=\frac{G(\omega)}{i \omega}=\eta \frac{i-\omega \lambda_{2}}{i-\omega \lambda_{1}}=\eta\left[\frac{\lambda_{2}}{\lambda_{1}}+i \frac{\lambda_{1}-\lambda_{2}}{\lambda_{1}\left(i-\omega \lambda_{1}\right)}\right],
$$

which is a simple proper frequency response function given that the number of poles equals the number of zeros. In the right-hand side of equation (46) we have separated the finite limiting value of the complex viscosity at the high frequency limit which when transformed in time domain it yields a singularity at the tie origin of the relaxation stiffness

$$
G(t)=\frac{\eta}{\lambda_{1}}\left[\lambda_{2} \delta(t-0)+G\left(1-\frac{\lambda_{2}}{\lambda_{1}}\right) e^{-\frac{t}{\lambda_{1}}}\right]
$$


Equation (47) is well known to the literature (Bird et al 1987 among others). Similar to the complex viscosity, the complex fluidity of the Jeffreys fluid is a simple proper frequency response function and its corresponding impulse strain-rate response function yields a singularity of the time origin. Table 2 summarizes all the basic frequency response and time response functions of the Poynting-Thomson solid and Jeffreys fluid examined in this paper. The original contribution of this paper is the addition of the Dirac delta functions marked with bold face in the complex viscosity of the Poynting-Thomson solid and the complex compliance of the Jeffreys fluid. Similarly, in bold face are marked in the corresponding time response functions the $1 / 2$ values resulting from the Fourier transform of the appended Dirac delta functions, which convert the non-causal signum functions $\frac{1}{2} \operatorname{sgn}(t)$ to the causal Heaviside

function $=\frac{1}{2}+\frac{1}{2} \operatorname{sgn}(t)=H(t)$.

\section{Conclusions}

In this paper the basic frequency response functions of the three-parameter PoyntingThomson solid and the three-parameter Jeffreys fluid are revisited. Each of these rheological models has either a dynamic complex viscosity or a dynamic complex compliance with an imaginary term that has a singularity at $\omega=0$. Using the properties of the Hilbert transform and the associated Kramers-Kronig relations it is showed that such frequency response functions should be corrected by adding a delta function in the real part. The presence of a Dirac delta function in the real part extends the concept of analyticity to generalized functions and essentially makes the corresponding frequency response functions well defined in the neighborhood $\omega=0$. This operation ensures causality of the corresponding time response functions which provide directly the stress or the strain histories via convolution integrals. 


\section{TABLE 2. Basic Frequency response and Time-Response Functions of the Three-Parameter Models.}

\begin{tabular}{|c|c|c|}
\hline & Three Parameter Poynting-Thomson Solid & Three Parameter Jeffreys fluid \\
\hline Constitutive Equation & $\tau(t)+\lambda_{1} \frac{d \tau(t)}{d t}=G\left[\gamma(t)+\lambda_{2} \frac{d \gamma(t)}{d t}\right]$ & $\tau(t)+\lambda_{1} \frac{d \tau(t)}{d t}=\eta\left[\frac{d \gamma(t)}{d t}+\lambda_{2} \frac{d^{2} \gamma(t)}{d t^{2}}\right]$ \\
\hline Complex Modulus: $G(\omega)$ & $\frac{G}{\lambda_{1}}\left[\lambda_{2}-\frac{\lambda_{2}-\lambda_{1}}{1+\omega^{2} \lambda_{1}^{2}}+i \frac{\omega \lambda_{1}\left(\lambda_{2}-\lambda_{1}\right)}{1+\omega^{2} \lambda_{1}^{2}}\right]$ & $\eta \omega \frac{i-\omega \lambda_{2}}{1+i \omega \lambda_{1}}$ \\
\hline Complex Compliance: $J(\omega)$ & $\frac{1}{G \lambda_{2}}\left[\lambda_{1}+\frac{\lambda_{2}-\lambda_{1}}{1+\omega^{2} \lambda_{2}^{2}}-i \frac{\omega \lambda_{2}\left(\lambda_{2}-\lambda_{1}\right)}{1+\omega^{2} \lambda_{2}^{2}}\right]$ & $\frac{\lambda_{1}-\lambda_{2}}{\eta}\left[\frac{1}{1+\lambda_{2}^{2} \omega^{2}}-i \frac{\omega \lambda_{2}}{1+\lambda_{2}^{2} \omega^{2}}\right]+\frac{\pi}{\eta}\left[\boldsymbol{\delta}(\boldsymbol{\omega}-\mathbf{0})-\frac{1}{\pi \omega}\right]$ \\
\hline Complex Viscosity, $\eta(\omega)$ & $G\left(\lambda_{2}-\lambda_{1}\right)\left[\frac{1}{1+\lambda_{1}{ }^{2} \omega^{2}}-i \frac{\omega \lambda_{1}}{1+\lambda_{1}{ }^{2} \omega^{2}}\right]+\pi G\left[\boldsymbol{\delta}(\boldsymbol{\omega}-\mathbf{0})-i \frac{1}{\pi \omega}\right]$ & $\frac{\eta}{\lambda_{1}}\left[\lambda_{2}+\frac{\lambda_{1}-\lambda_{2}}{1+\omega^{2} \lambda_{1}^{2}}-i \frac{\omega \lambda_{1}\left(\lambda_{1}-\lambda_{2}\right)}{1+\omega^{2} \lambda_{1}^{2}}\right]$ \\
\hline Complex Fluidity, $\varphi(\omega)$ & $\frac{i \omega}{G} \frac{1+i \omega \lambda_{1}}{1+i \omega \lambda_{2}}$ & $\frac{1}{\eta \lambda_{2}}\left[\lambda_{1}-\frac{\lambda_{1}-\lambda_{2}}{1+\omega^{2} \lambda_{2}^{2}}+i \frac{\omega \lambda_{2}\left(\lambda_{1}-\lambda_{2}\right)}{1+\omega^{2} \lambda_{2}^{2}}\right]$ \\
\hline Memory Function, $q(t)$ & $\frac{G}{\lambda_{1}}\left[\lambda_{2} \delta(t-0)-\left(\frac{\lambda_{2}}{\lambda_{1}}-1\right) e^{-\frac{t}{\lambda_{1}}}\right]$ & $\frac{\eta}{\lambda_{1}}\left[\lambda_{2} \frac{d \delta(t-0)}{d t}+\left(1-\frac{\lambda_{2}}{\lambda_{1}}\right) \delta(t-0)-\frac{1}{\lambda_{1}}\left(1-\frac{\lambda_{2}}{\lambda_{1}}\right) e^{-\frac{t}{\lambda_{1}}}\right]$ \\
\hline Retardation Fluidity, $\varphi(t)$ & $\frac{1}{G \lambda_{2}}\left[\lambda_{1} \delta(t-0)+\left(1-\frac{\lambda_{1}}{\lambda_{2}}\right) e^{-\frac{t}{\lambda_{2}}}\right]$ & $\frac{1}{\eta}\left[\frac{\mathbf{1}}{\mathbf{2}}+\frac{1}{2} \operatorname{sgn}(t)+\left(\frac{\lambda_{1}}{\lambda_{2}}-1\right) e^{-\frac{t}{\lambda_{2}}}\right]$ \\
\hline Relaxation Modulus, $G(t)$ & $G\left[\frac{\mathbf{1}}{\mathbf{2}}+\frac{1}{2} \operatorname{sgn}(t)\right]+G\left(\frac{\lambda_{2}}{\lambda_{1}}-1\right) e^{-\frac{t}{\lambda_{1}}}$ & $\frac{\eta}{\lambda_{1}}\left[\lambda_{2} \delta(t-0)+G\left(1-\frac{\lambda_{2}}{\lambda_{1}}\right) e^{-\frac{t}{\lambda_{1}}}\right.$ \\
\hline Impulse Strain-Rate Response Function, $\psi(t)$ & $\frac{1}{G \lambda_{2}}\left(\lambda_{1} \frac{d \delta(t-0)}{d t}+\left(1-\frac{\lambda_{1}}{\lambda_{2}}\right) \delta(t-0)-\frac{1}{\lambda_{2}}\left(1-\frac{\lambda_{1}}{\lambda_{2}}\right) e^{-\frac{t}{\lambda_{2}}}\right)$ & $\frac{1}{\eta \lambda_{2}}\left(\lambda_{1} \delta(t-0)-\left(\frac{\lambda_{1}}{\lambda_{2}}-1\right) e^{-\frac{t}{\lambda_{2}}}\right)$ \\
\hline
\end{tabular}




\section{References}

Bird, B., Armstrong, R., and Hassager, O., Dynamic of polymeric liquids (Wiley \& Sons, New York, 1987).

Bode, H. W., Network analysis and feedback amplifier design (Van Nostrand Reinhold, Princeton, N.J., 1959).

Booij, H.,C., and Thoone, G., P., J., M., "Generalization of Kramers-Kronig transforms and some approximations of relations between viscoelastic quantities.", Rheologica Acta, 21, 15-24 (1982).

Caracciolo, R., Gasparetto, A., Giovagnoni, M., "Application of causality check and of reduced variables method for experimental determination of Young's modulus of a viscoelastic material.”, Mechanics of Materials, 33, 693-703 (2001).

Dhont, J., K., G., Wagner, N., J., “Superposition rheology.”, Physical Review, 63, $021406(2001)$.

Dirac, P. A. M., The principles of quantum mechanics (Oxford University Press, Oxford, U.K., 1958).

Everaers, R., Sukumaran, S., K., Grest, G., S., Svaneborg, C., Sivasubramanian, A., and Kremer, K., "Rheology and Microscopic Topology of Entangled Polymer Liquids.", Science, 303, 823-826 (2004).

Fannin, P., C., Molina, A., and Charles, S., W., "On the generation of complex susceptibility data through the use of the Hilbert transform.”, J. Phys., 26, 2006-2009 (1993).

Ferry, J.D., Viscoelastic Properties of Polymers (Wiley, New York, 1980).

Giesekus, H., "An alternative approach to the linear theory of viscoelasticity and some characteristic effects being distinctive of the type of material," Rheologica Acta, 34, 2-11 (1995).

Harris, C. M. (ed.) Shock and vibration handbook (McGraw-Hill, New York, 1988).

Kothandapani, M., Srinivas, S., "Peristaltic transport of a Jeffrey fluid under the effect of magnetic field in an asymmetric channel", International Journal of Non-Linear Mechanics, 43, 915-924 (2008). 
Kytopoulos, V., N., Bourkas, G., D., Sideridis, E., "Some New Operational Modes and Parameters of Stress Relaxation for the Viscoelastic Characterization of Solid Polymers. III. The Area Ratio Mode and the Intrinsic "Strain-Clock” Function.”, J. of Applied Polymer Science, 87, 149-158 (2003).

Lebon, G., Parmentier, P., Teller, O., Dauby, P., C., "Benard-Marangoni instability in a visoelastic Jeffreys' fluid layer", Rheologica Acta, 33, 257-266 (1994).

Lighthill, M. J. An introduction to Fourier analysis and generalized functions (Cambridge University Press, Cambridge, U.K., 1989).

Makris, N., "Stiffness, Flexibility, Impedance, Mobility, and Hidden Delta Function", J.Engrg.Mech., ASCE, 123(11), 1202-1208, (1997).

Manos, T., Merinakis, G., Tsangaris, S., "Oscillating viscoelastic flow in a curved duct- Exact analytical and numerical solution", J. Non-Newtonian Fluid Mech., 135, 8-15 (2006).

Mezerova, L., "Experimental Verification of Viscoelastic Properties of Spongy Bone Tissue", 7, 45-46 (2004).

Morse, P. M., and Feshbach H., Methods of theoretical physics (McGraw-Hill, New York, 1953).

Parot, J.-M., Duperray, B., “Applications of exact causality relationships to material dynamic analysis.”, Mechanics of Materials, 39, 419-433 (2007).

Pipkin, A. C. Lectures on Viscoelasticity Theory. Second Edition (Springer, New York, 1986).

Pritz, T., "Unbounded complex modulus of viscoelastic materials and the KramersKronig relations.”, J. Sound and Vibration, 279, 687-697 (2005).

Rohrs, C. E., Melsa, J. L., and Scults, D. G., Linear control systems (McGraw-Hill, New York, 1993).

Tschoegl, N.,W., The Phenomenological Theory of Linear Viscoelastic Behavior. An Introduction (Springer, Berlin, 1989).

Veletsos, A. S., and Verbic, B., "Basic response functions for elastic foundations," J.Engrg.Mech., ASCE, 100(2), 189-202, (1974). 
Vlassopoulos D., "Commentary on the observations of solid-like rheological response in unentangled polymer melts by H.Mendil, P.Baroni, L.Noirez., D.Collin, P.Martinoty.”, Eur. Phys. J. E, 19, 113-117 (2006).

Zhifa, Y., Zhiyin, W., Luqing, Z., Ruiguang, Z., Nianxing, X., "Back-analysis of viscoelastic displacements in a soft rock road tunnel.”, International Journal of Rock Mechanics \& Mining Sciences, 38, 331-341 (2001). 


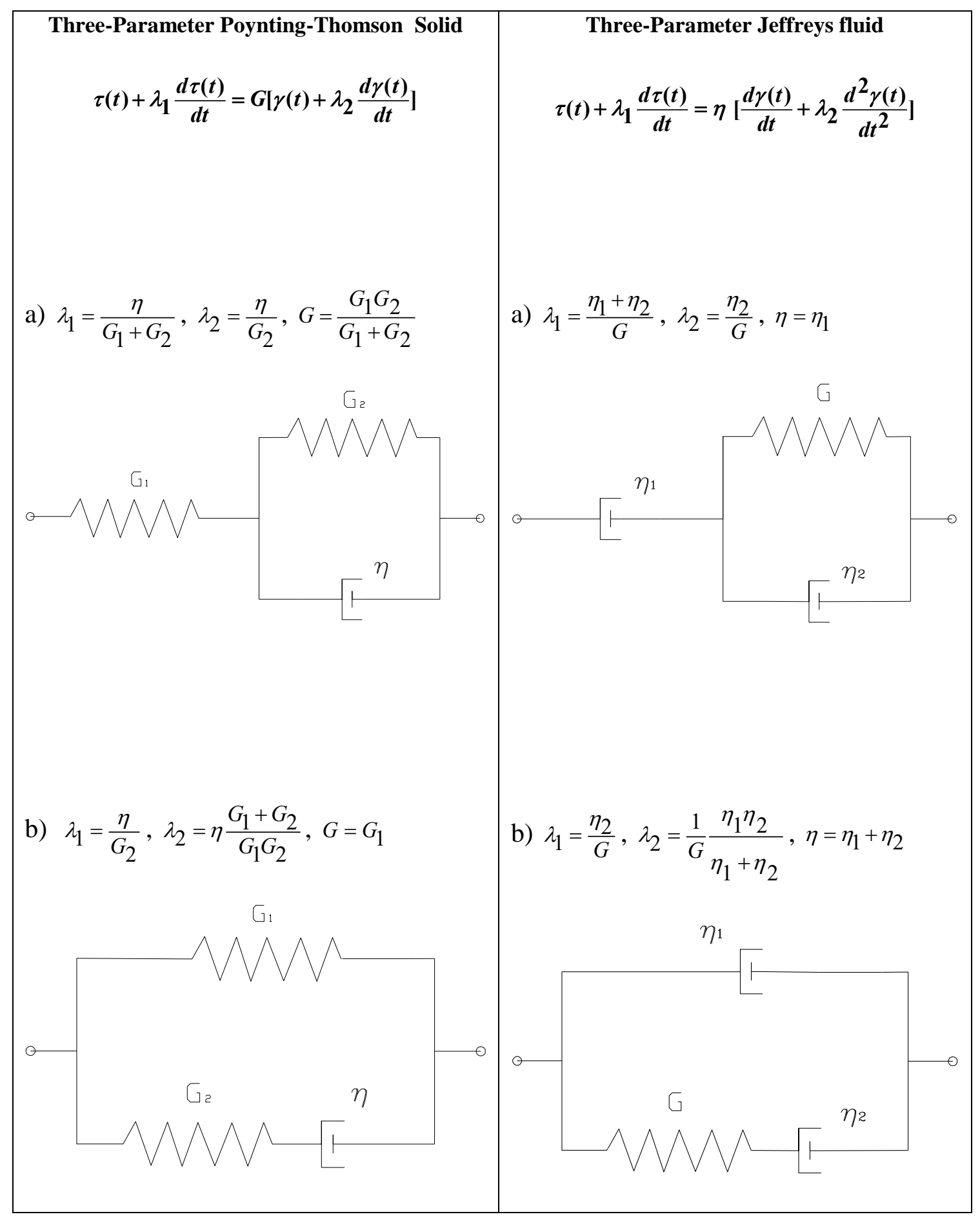

Figure 1. Alternative configuration of the three-parameter Poynting-Thomson solid (left) and the threeparameter Jeffreys fluid (right). 